\title{
Efficacy of Acetylshikonin in Preventing Obesity and Hepatic Steatosis in $\mathrm{db} / \mathrm{db}$ Mice
}

\author{
Mei-Ling Su, Yu He, Qi-Sen Li and Bang-Hao Zhu * \\ Department of Pharmacology, Cardiac and Cerebral Vascular Research Center, Zhongshan School of Medicine, \\ Sun Yat-sen University, No. 74 Zhongshan Rd. 2, Guangzhou 510080, China; sumeiling124@163.com (M.-L.S.); \\ 13760686022@163.com (Y.H.); XY_Sen@hotmail.com (Q.-S.L.) \\ * Correspondence: zhubh@mail.sysu.edu.cn; Tel.: +86-20-8733-4786
}

Academic Editor: Min-Hsiung Pan

Received: 24 June 2016; Accepted: 25 July 2016; Published: 28 July 2016

\begin{abstract}
Zicao (Lithospermum erythrorhizon) has been used in clinics as a traditional Chinese medicine for thousands of years. Acetylshikonin (AS) is the main ingredient of Zicao, Xinjiang, China. The objective of this study was to investigate the anti-obesity and anti-nonalcoholic fatty liver disease (NAFLD) efficacy of AS in a model of spontaneous obese $\mathrm{db} / \mathrm{db}$ mice. Mice were divided into Wild Type (WT) groups and $\mathrm{db} / \mathrm{db}$ groups, which received no treatment or treatment with $100 \mathrm{mg} / \mathrm{kg} /$ day clenbuterol (CL) hydrochloride or $540 \mathrm{mg} / \mathrm{kg} /$ day AS by oral gavage for eight weeks. The results provided the evidence that AS prevented obesity and NAFLD including reduction in body weight, food efficiency ratio, serum triglyceride (TG) and free fatty acid (FFA) levels in $\mathrm{db} / \mathrm{db}$ mice. Administration of AS markedly suppressed the levels of hepatic alanine aminotransferase (ALT), aspartate aminotransferase (AST) and pro-inflammatory cytokines in treated groups when compared with that of $\mathrm{db} / \mathrm{db}$ groups. Further investigation of the lipid synthesis-related protein using Western blotting revealed that hepatic protein expression of sterol regulatory element-binding protein-1 (SREBP-1), fatty acid synthetase (FAS) and 3-hydroxy-3-methylglutaryl-coenzyme A reductase (HMGCR) were significantly downregulated by AS treatment. These findings suggest that AS exerts anti-obesity and anti-NAFLD effects through the regulation of lipid metabolism and anti-inflammatory effects.
\end{abstract}

Keywords: acetylshikonin; obesity; nonalcoholic fatty liver disease; lipid metabolism; inflammatory cytokines

\section{Introduction}

Obesity is an increasing risk factor of various metabolic diseases, including type 2 diabetes mellitus (T2DM), insulin resistance, hyperlipidemia, and NAFLD [1]. Obesity-related NAFLD is a clear risk factor of death in the general population around the world. NAFLD has typical characteristics with ectopic fat accumulation in hepatocytes. The ectopic fat accumulation can impair hepatic function, developing into nonalcoholic steatohepatitis (NASH), cirrhosis, and liver cancer [2,3]. Estimates of NAFLD prevalence were approximately $20 \%-25 \%$ worldwide; and the prevalence incidence of NAFLD was much higher in people with obesity than in normal populations [4].

At the first stage of NAFLD, fatty liver is characterized by ectopic fat droplet accumulation in hepatocytes and meanwhile is associated with elevation of liver enzyme levels such as ALT and AST, which is reversible. Hormone-sensitive lipase (HSL), adipose TG lipase (ATGL), and perilipin are crucial enzymes of lipolysis that catalyze the intracellular triacylglycerol to release glycerol and non-esterified fatty acid [5,6]. Lipid metabolism contains lipolysis and lipid synthesis. SREBP-1 plays an essential role in modulating the expression of lipid synthesis-related enzymes [7]. Studies have been 
reported that SREBP-1 played an important role in the fat droplet infiltration of fatty liver, indicating the expression of SREBP-1 was significantly increased in NAFLD [8].

In the second stage of NAFLD, fatty liver turns into a steatohepatitis, which is accompanied by inflammatory reaction [9]. Evidence manifests that obesity is closely associated with the development of inflammatory in liver diseases including NAFLD and NASH [10]. Although the NAFLD progression is complex and remains enigmatic, several pro-inflammatory cytokines are important regulatory factors in NAFLD. Various pro-inflammatory cytokines, such as tumour necrosis factor-a (TNF- $\alpha$ ), interleukin-6 (IL-6) and interleukin-1 $\beta$ (IL-1 $\beta$ ) contribute to steatohepatitis development and progression [11-13]. Therefore, it is a promising target in the prevention and treatment of NAFLD through inhibiting pro-inflammatory cytokines production and reducing inflammatory reaction.

Although studies have revealed that some pharmacological agents exert beneficial effects on rat models of NAFLD, none have shown promise for reversing NAFLD so far. Therefore, the research and development of an efficient anti-NAFLD drug is urgent and desired. Zicao has been used in traditional herbal medicine for thousands years in China, and its primary active ingredients are naphthoquinone derivatives containing AS (Figure 1), shikonin, $\beta$-hydroxyisovaleryshikonin, deoxyshikonin, $\beta, \beta$-dimethylacrylshikonin and isovalerylalkannin [14]. Its derivatives have revealed various pharmacological activities including anti-tumorigenic, anti-bacterial, anti-viral, anti-inflammatory, and wound healing bioactivity $[15,16]$. Zicao was obtained from Xinjiang in China and AS is the main ingredient of Zicao. In addition, previous study has reported that arnebin-1 (naphthoquinone derivative of Zicao) accelerated the wound healing process in diabetic rats. Moreover, our previous studies have demonstrated that AS could prevent obesity in rats on a high-fat diet model [17]. These suggest that AS will become a beneficial and valuable agent. The present study aimed to investigate the effect of it in preventing or treating obesity and NAFLD in a model of $\mathrm{db} / \mathrm{db}$ mice, and further clarify its underlying molecular mechanisms.

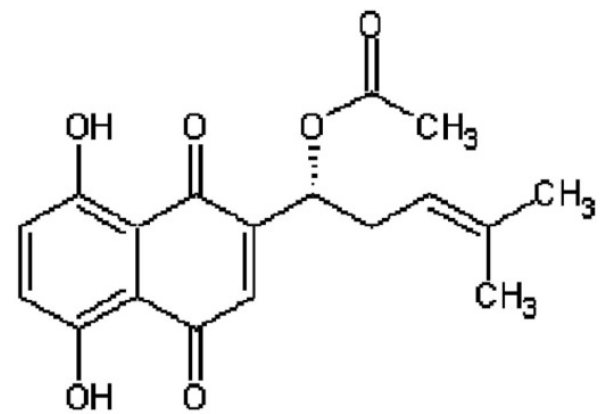

Figure 1. Chemical structures of acetylshikonin.

\section{Results}

\subsection{AS Suppressed Obesity in $d b / d b$ Mice}

Eight-week-old male C57BL/6J mice were divided into four groups: WT control; obese $\mathrm{db} / \mathrm{db}$ control; CL treatment (100 mg/ kg/day) and AS treatment $(540 \mathrm{mg} / \mathrm{kg} /$ day). The food intake and body weight of the mice were measured weekly during the experimental period. As shown in Figure 2A, weekly food intake per mouse was not different among all groups during the experimental period $(p>0.05)$. Eight-week administration of CL and AS suppressed the average weight gain by $36.6 \%$ and $54.4 \%$ vs. $\mathrm{db} / \mathrm{db}$ group $p<0.05$, respectively (Figure $2 \mathrm{C}, \mathrm{D}$ ). Moreover, the food efficiency ratio was significantly decreased in CL and AS treated groups by $33.7 \%$ and $48.2 \%$ when compared with that of obese $\mathrm{db} / \mathrm{db}$ group (Figure $2 \mathrm{~B}$ ). There were not any pathological signs in the mice during the experimental period. These results demonstrate that both CL and AS efficiently reduce the weight gain in $\mathrm{db} / \mathrm{db}$ mice. 
A

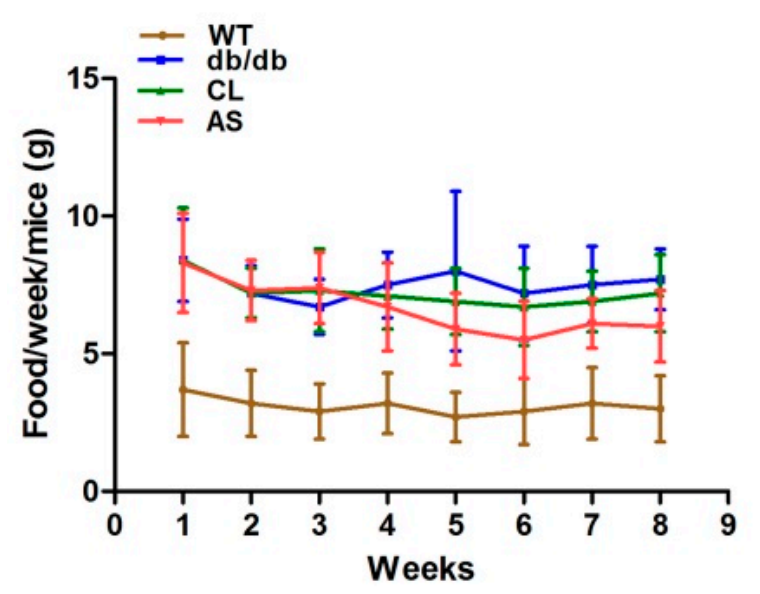

C

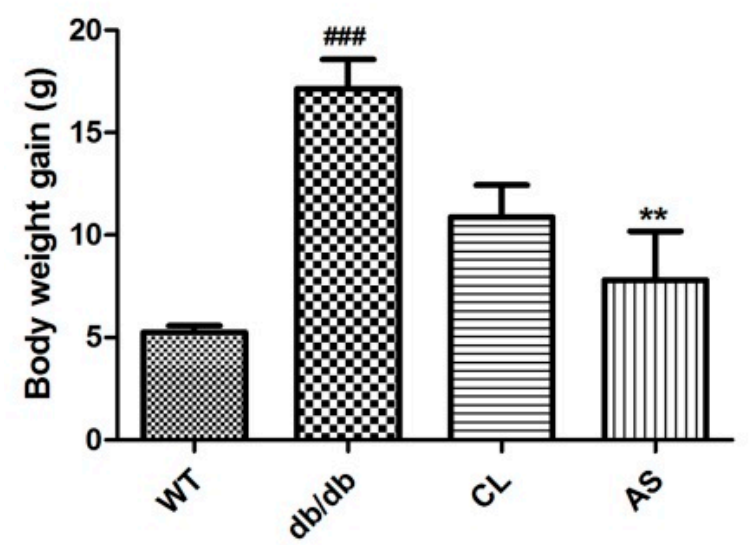

B
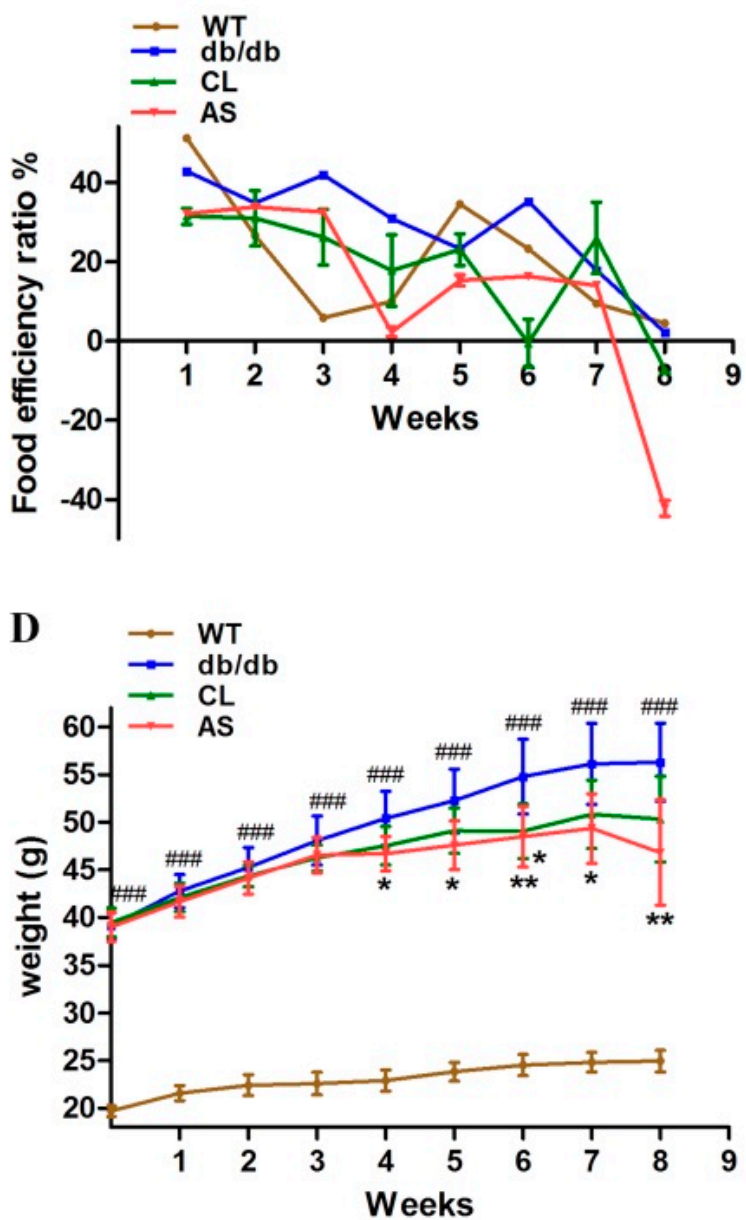

Figure 2. AS suppressed weight gain in $\mathrm{db} / \mathrm{db}$ mice. Mice were divided into four groups: WT control; obese $\mathrm{db} / \mathrm{db}$ control; CL treatment (100 mg/kg/day) and AS treatment ( $540 \mathrm{mg} / \mathrm{kg} /$ day). The drugs were oral gavage for eight weeks. (A) food intake was recorded weekly; (B) food efficiency ratio was calculated as body weight gain divided by food intake; (C) change in body weight was measured weekly; and (D) body weight gain was measured weekly. Values are expressed as mean \pm SEM $(n=24)$. ${ }^{*} p<0.05,{ }^{* *} p<0.01$ vs. db/db group; ${ }^{\# \# \# ~} p<0.001$ vs. WT group.

\subsection{Effect of AS on Abdominal Adiposity in db/db Mice}

Mice images were acquired by a digital camera at the end of the experiment (Figure 3A). For the determination of abdominal adiposity, we performed micro-CT imaging to assess the effect of CL and AS; Figure 3B showed that body fat was reduced by CL and AS treatment (WT group, $941.08 \mathrm{~mm}^{3}$; $\mathrm{db} / \mathrm{db}$ group, 15,896.82 $\mathrm{mm}^{3}$; CL group, 13,452.87 $\mathrm{mm}^{3}$, AS group, 13,184.78 $\mathrm{mm}^{3}$; respectively). In addition, the body mass index (BMI) of obese $\mathrm{db} / \mathrm{db}$ mice was 2.2-fold higher than that of the WT group, while it was decreased by $15.4 \%$ and $17.1 \%$ in $\mathrm{db} / \mathrm{db}$ mice with CL and AS treated groups, when compared with obese $\mathrm{db} / \mathrm{db}$ group $(p<0.05 ; n=6$; Figure $3 \mathrm{C})$. Fat volume in obese $\mathrm{db} / \mathrm{db}$ mice was 16.3-fold higher than that of the WT group. It was significantly reduced by $8.8 \%$ and $14.9 \%$ in CL and AS treated groups when compared with the obese $\mathrm{db} / \mathrm{db}$ group $(p<0.05 ; n=6$; Figure 3D). 


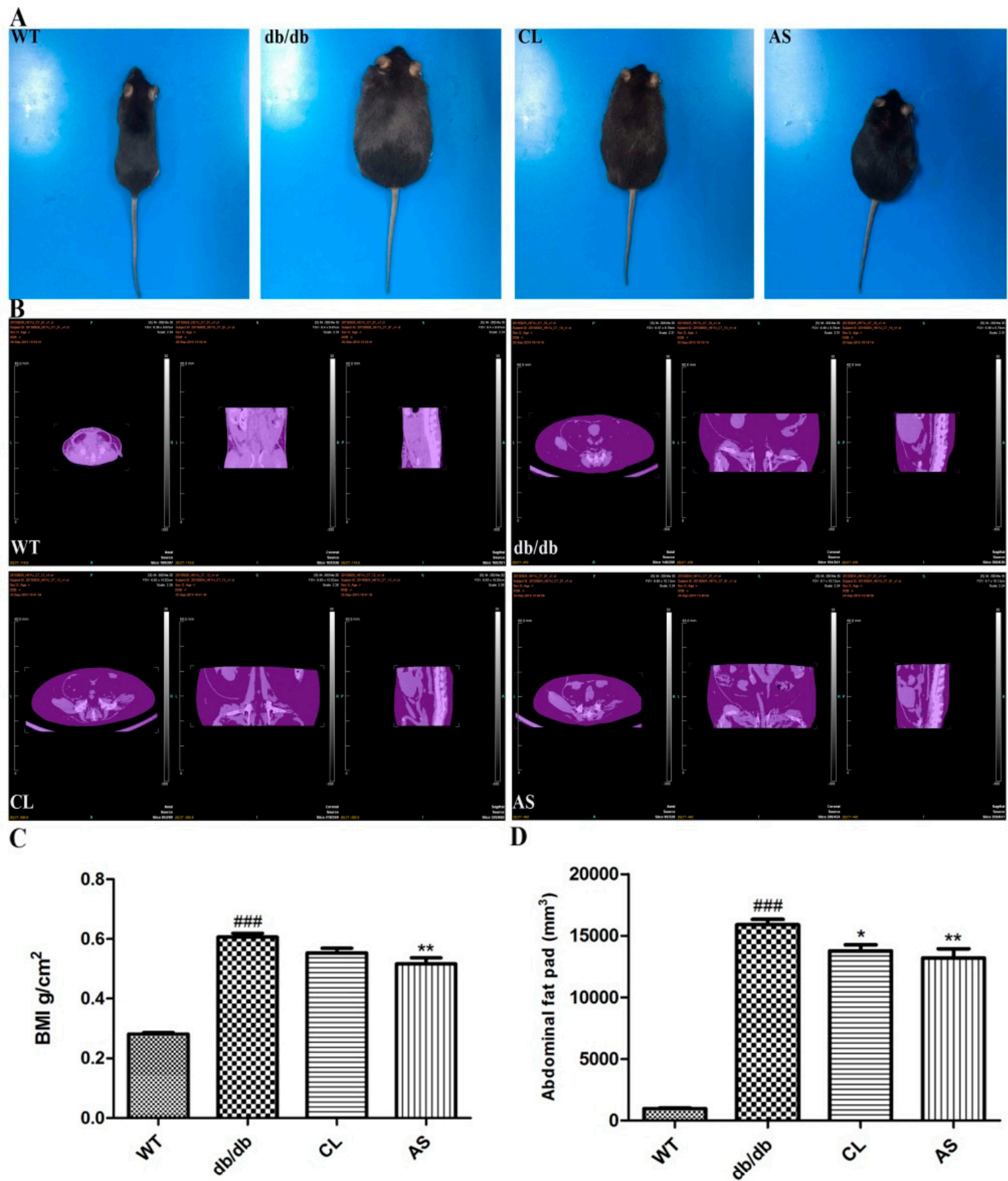

Figure 3. Effect of AS on abdominal adiposity in $\mathrm{db} / \mathrm{db}$ mice. (A) mice images were acquired by a digital camera at the end of experiment; (B) micro-computed tomography (CT) images of abdominal fat of mice in $\mathrm{WT}, \mathrm{db} / \mathrm{db}, \mathrm{CL}$, or AS group; (C) BMI = weight (g)/length/length $\left(\mathrm{cm}^{2}\right)$; (D) fat volumes $\left(\mathrm{mm}^{3}\right)$ in mice are shown. The values represent the mean $\pm \operatorname{SEM}(n=6) .{ }^{*} p<0.05,{ }^{* *} p<0.01 \mathrm{vs} . \mathrm{db} / \mathrm{db}$ group; ${ }^{\# \#} p<0.001$ vs. WT group.

\subsection{AS Reduced Adipose Tissue, Liver Weight and Hepatic Lipid Accumulation in db/db Mice}

Administration of CL and AS markedly suppressed the increase in the amount of epididymal, perirenal fat pads, and liver weight ( $p<0.05$; Figure $4 \mathrm{~A}, \mathrm{~B})$. The hepatic TG level of $\mathrm{db} / \mathrm{db}$ mice was 2.03 -fold higher than that of the WT group, while it was markedly decreased by $14.4 \%$ and $28.9 \%$ in $\mathrm{CL}$ and AS treated groups when compared with the obese $\mathrm{db} / \mathrm{db}$ group $(p<0.05 ; n=6$; Figure $4 \mathrm{C})$. 
The pathological signs, including morphological and histological changes in the adipose tissue and liver, were ameliorated in the CL and AS treated groups (Figure 4D,E). Furthermore, the deposition of lipid droplets in the hepatocytes was significantly inhibited in the CL and AS treated groups (Figure 4F) as detected by Oil Red $\mathrm{O}$ staining. These results suggest that AS reduces adipose tissue mass and prevents fat accumulation in the liver of $\mathrm{db} / \mathrm{db}$ mice.

A

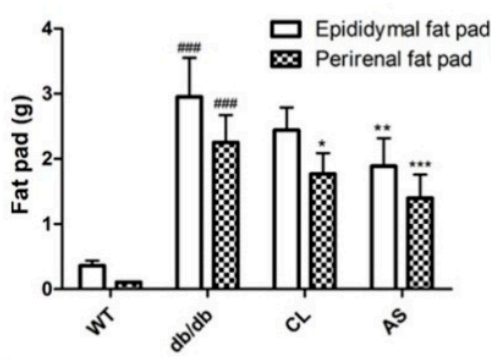

B

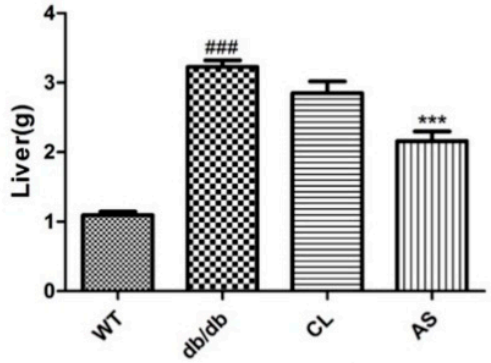

C

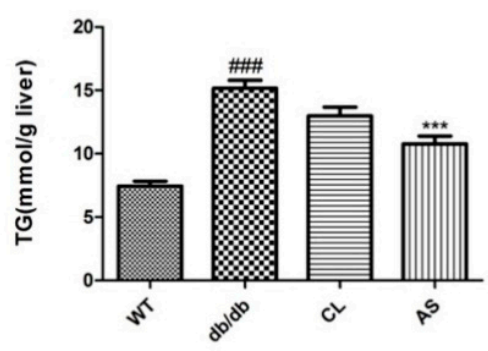

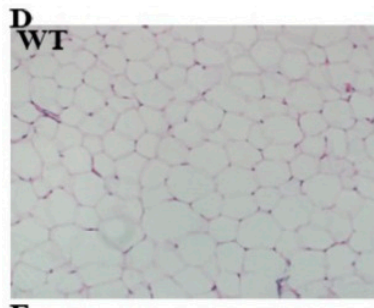

E
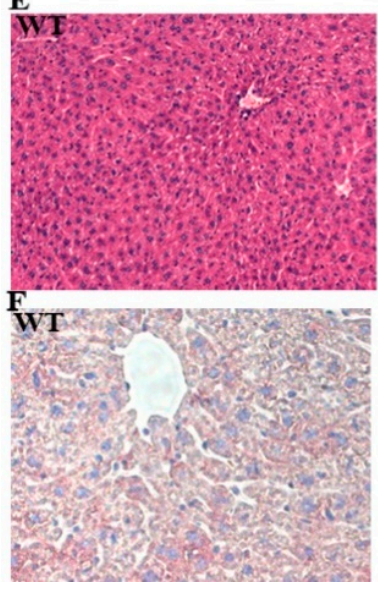
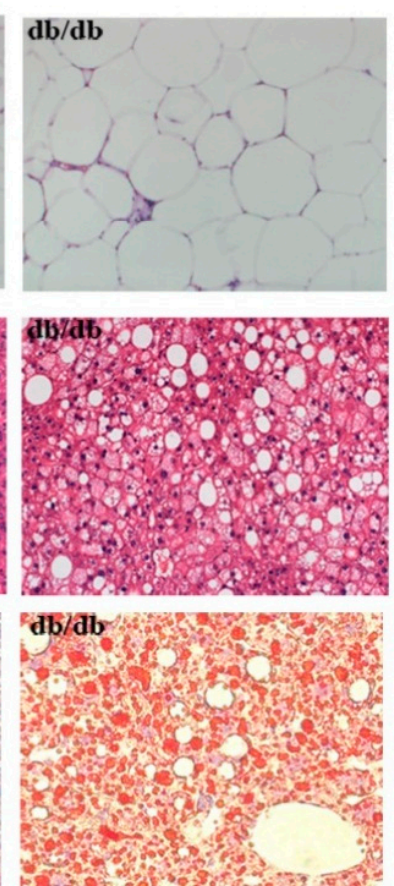
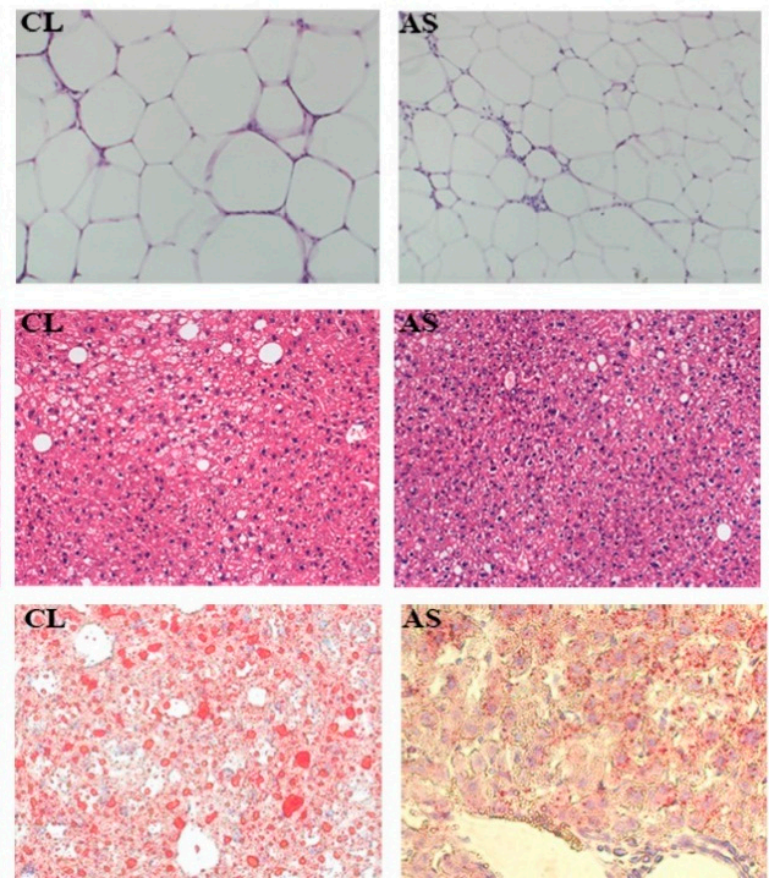

Figure 4. AS reduced adipose tissue, liver weight and hepatic lipid accumulation in $\mathrm{db} / \mathrm{db}$ mice. (A) epididymal and perirenal fat pads; (B) liver weight; (C) hepatic TG was measured after mice had fasted for $12 \mathrm{~h}$ at the end of experiment; (D) epididymal adipose tissue and (E) liver were stained with hematoxylin and eosin; and (F) liver was stained with Oil Red $\mathrm{O}$ to detect the quantities of lipids. Images were acquired at $200 \times$ magnification by a microscopy. Values are expressed as mean \pm SEM $(n=6) .{ }^{*} p<0.05,{ }^{* *} p<0.01,{ }^{* * *} p<0.001$ vs. db/db group; ${ }^{\# \#} p<0.001$ vs. WT group.

\subsection{AS Decreased the Blood Lipids and Hepatic Enzyme Levels in db/db Mice}

Table 1 shows that the plasma biochemical levels of TG, FFA, CHE, AST, ALT, and glucose were significantly increased in obese $\mathrm{db} / \mathrm{db}$ group compared with that of the WT group. However, plasma TG and FFA levels were decreased by $56.8 \%$ and $46.0 \%$ in $\mathrm{db} / \mathrm{db}$ mice treated with AS, respectively, relative to obese $\mathrm{db} / \mathrm{db}$ group $(p<0.01 ; n=6)$. Similarly, the plasma FFA level was reduced by $40.2 \%$ in $\mathrm{db} / \mathrm{db}$ mice treated with $\mathrm{CL}$, as compared with obese $\mathrm{db} / \mathrm{db}$ group $(p<0.01)$. Meanwhile, the plasma glucose, CHE, AST and ALT levels of $\mathrm{db} / \mathrm{db}$ mice were 4.9-, 2.0-, 1.6- and 4.5-fold higher than that of WT group, respectively. Administration of AS plasma glucose, CHE, AST and ALT levels were 
notably decreased by $34.1 \%, 45.5 \%, 27.2 \%$ and $45.8 \%$ in $\mathrm{db} / \mathrm{db}$ mice, respectively, relative to obese $\mathrm{db} / \mathrm{db}$ group $(p<0.05 ; n=6)$. These results show that obesity and NAFLD are ameliorated in the blood lipids and hepatic enzyme levels by AS treatment.

Table 1. AS reduced the blood lipids and hepatic enzyme levels in $\mathrm{db} / \mathrm{db}$ mice.

\begin{tabular}{ccccc}
\hline Parameter & WT & db/db & CL & AS \\
\hline TG $(\mathrm{mM})$ & $0.65 \pm 0.13$ & $1.92 \pm 0.63^{\# \#}$ & $1.82 \pm 0.75$ & $0.83 \pm 0.15^{* *}$ \\
FFA $(\mathrm{mM})$ & $0.58 \pm 0.07$ & $1.26 \pm 0.19^{\# \#}$ & $0.76 \pm 0.20^{* *}$ & $0.68 \pm 0.11^{* *}$ \\
Glucose $(\mathrm{mM})$ & $5.72 \pm 1.01$ & $27.80 \pm 4.07^{\# \#}$ & $25.75 \pm 5.96$ & $18.32 \pm 2.63^{* *}$ \\
CHE(mM) & $3.40 \pm 0.45$ & $6.89 \pm 0.57^{\# \#}$ & $6.63 \pm 0.73$ & $3.76 \pm 0.37^{* *}$ \\
AST(IU/L) & $73.63 \pm 8.33$ & $116.42 \pm 22.59^{\# \#}$ & $112.43 \pm 23.18$ & $84.78 \pm 12.23^{*}$ \\
ALT(IU/L) & $20.82 \pm 3.15$ & $94.48 \pm 24.90^{\# \#}$ & $99.02 \pm 39.22$ & $51.170 \pm 14.74^{*}$ \\
\hline
\end{tabular}

TG, triglyceride; FFA, free fatty acid; CHE, cholinesterase; AST, alanine aminotransferase; ALT, alanine aminotransferase. Values are expressed as mean \pm SEM of $5 \sim 6$ mice. ${ }^{*} p<0.05,{ }^{* *} p<0.01 \mathrm{vs}$. db/db group; ${ }^{\# \#} p<0.01$ vs. WT group.

\subsection{AS Increased the Expressions of Lipid-Metabolizing Enzymes in Adipose Tissue}

Perilipin, ATGL and HSL protein levels were markedly reduced in the obese $\mathrm{db} / \mathrm{db}$ group compared with that of the WT group; however, they were increased by 3.93-, 2.91-, and 2.66-fold in the CL treated group when compared with the obese $\mathrm{db} / \mathrm{db}$ group, respectively. In the case of the AS group, they were increased by 5.18-, 3.74-, and 3.58-fold, respectively. $(p<0.05 ; n=5$; Figure 5). These data indicate that AS increases key lipid-metabolizing enzymes in adipose tissue.
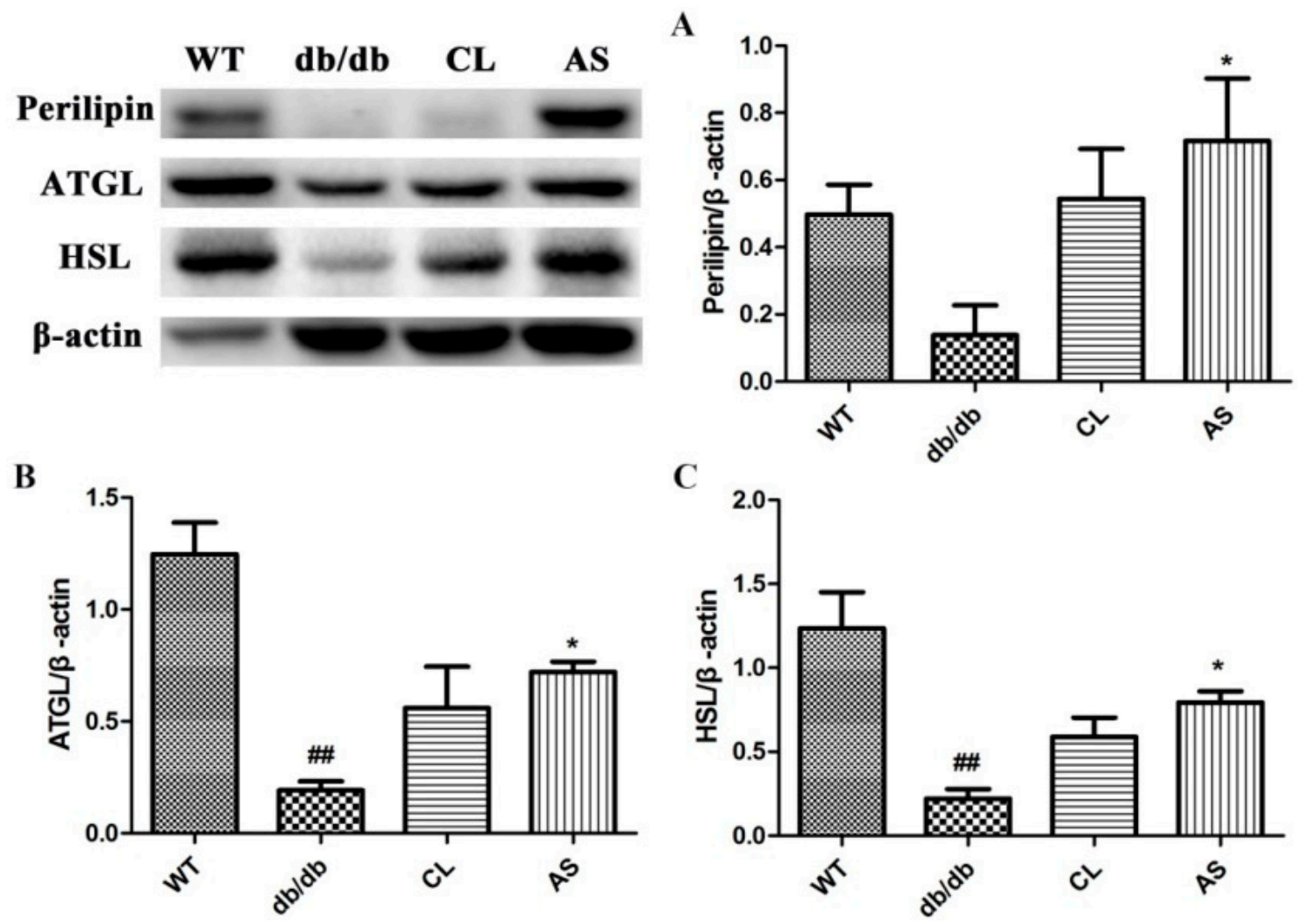

Figure 5. AS increased the expressions of lipid-metabolizing enzymes in adipose tissue. (A) perilipin; (B) ATGL; and (C) HSL protein expression in adipose tissue was analyzed by Western blot. Immunoblots are representative of five independent experiments; $\beta$-actin served as a loading control. Values are expressed as mean \pm SEM of five mice. ${ }^{*} p<0.05$ vs. the $\mathrm{db} / \mathrm{db}$ group; ${ }^{\# \#} p<0.01 \mathrm{vs}$. the WT group. 
2.6. AS Inhibited the Lipid Synthesis-Related Protein Expressions (SREBP-1, FAS and HMGCR) in the Liver

The hepatic protein expression levels of SREBP-1, FAS and HMGCR were markedly increased in the obese $\mathrm{db} / \mathrm{db}$ group compared with that of the WT group. Whereas SREBP-1, FAS and HMGCR protein levels were markedly downregulated by $58.8 \%, 61.7 \%$ and $56.2 \%$ in the AS treated group, respectively, as compared with that of the obese $\mathrm{db} / \mathrm{db}$ group $(p<0.05 ; n=5$; Figure 6$)$. These data indicate that AS can suppress TG synthesis in the liver.

\section{$\begin{array}{llll}\text { WT } & \text { db/db } & \text { CL } & \text { AS }\end{array}$}

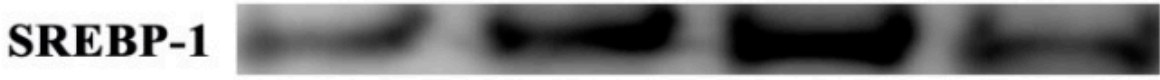

GAPDH

HMGCR

GAPDH

FAS

GAPDH

A

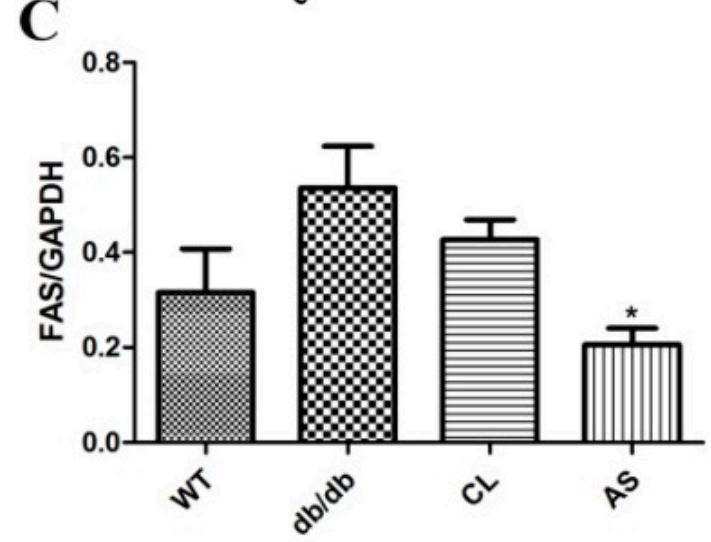

B

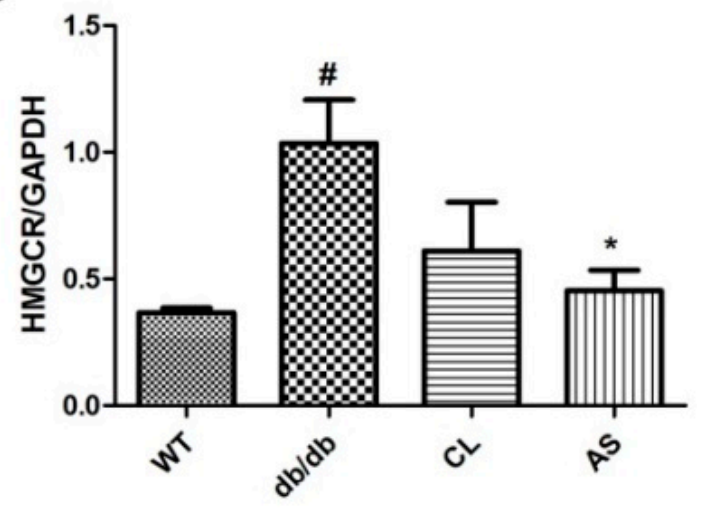

Figure 6. AS inhibited the lipid synthesis-related protein expressions in liver. (A) SREBP-1 and (B) HMGCR; (C) FAS protein expression in liver was analyzed by Western blot. Immunoblots are representative of five independent experiments; GAPDH served as a loading control. Values are expressed as mean \pm SEM of five mice. ${ }^{*} p<0.05,{ }^{* * *} p<0.001$ vs. $\mathrm{db} /$ db group; ${ }^{\#} p<0.05,{ }^{\# \#} p<0.01$ vs. WT group. 


\subsection{Effect of AS on Serum Pro-Inflammatory Cytokines in db/db Mice}

As shown in Figure 7, serum TNF- $\alpha$, IL-6 and IL-1 $\beta$ levels in obese $\mathrm{db} / \mathrm{db}$ group were increased by 3.75-, 4.61- and 4.39-fold when compared with that of the WT group, whereas administration of AS markedly suppressed the increase in the levels of serum pro-inflammatory cytokines by $49.1 \%$, $41.1 \%$ and $45.6 \%$, respectively, when compared with that of the obese $\mathrm{db} / \mathrm{db}$ group. These results demonstrate that AS exerts the regulation effect of the hepatic inflammation.

A

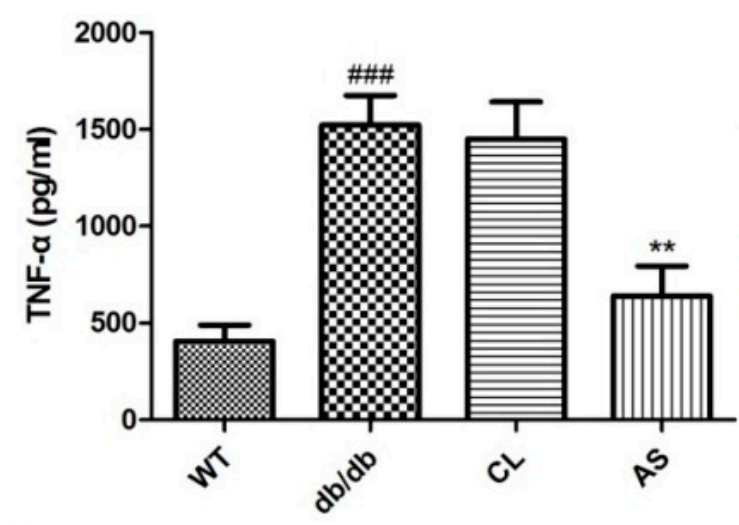

C

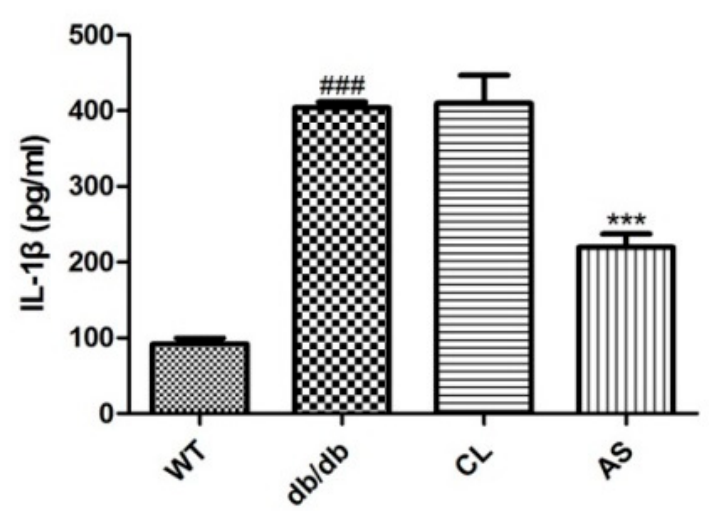

B

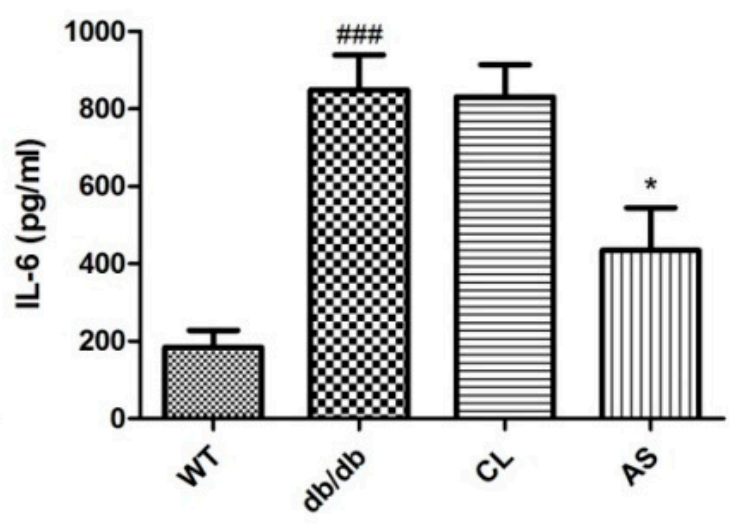

Figure 7. Effect of AS on pro-inflammatory cytokines in $\mathrm{db} / \mathrm{db}$ mice. Serum (A) TNF- $\alpha$; (B) IL-1 $\beta$ and (C) IL-6 levels were quantified using enzyme-linked immunosorbent assay (ELISA) kits, following the protocol recommended by the manufacturer. Values are expressed as mean \pm SEM of $5 \sim 6$ mice. ${ }^{*} p<0.05,{ }^{* *} p<0.01,{ }^{* * *} p<0.001$ vs. db/db group; ${ }^{\# \# \# ~} p<0.001$ vs. the WT group.

\section{Experimental Section}

\subsection{Reagents and Antibodies}

Traditional herbal Zicao were purchased from Xinjiang in China. TNF- $\alpha$, IL-1 $\beta$, and IL-6 ELISA Kits and anti- $\beta$-actin and anti-glyceraldehyde-3-phosphate dehydrogenase (GAPDH) were purchased from Boster Co. (Wuhan, China). Liver lipid content (A110-1 Triglycerides Assay kit; $100 \mu \mathrm{L}$ ) was purchased from Jiancheng Bioengineering Co. (Nanjing, China). Antibodies against HSL (Ser563), ATGL and horseradish peroxidase (HRP)-conjugated anti-rabbit secondary antibody were purchased from Cell Signaling Technology (Beverley, MA, USA). Antibodies against perilipin, SREBP-1, FAS and HMGCR were obtained from Abcam (Cambridge, UK). All other reagents were purchased from Sigma-Aldrich (St. Louis, MO, USA) unless otherwise specified. 


\subsection{Mice and Experimental Design}

Mice experiments were performed in accordance with the Guidelines and were approved by the Animal Research Center of Sun Yat-sen University. Male wild-type C57BLKS mice and C57BLKS/Lepr ${ }^{\mathrm{db}}(\mathrm{db} / \mathrm{db})$ mice were purchased from the Model Animal Research Center of Nanjing University (MARC, Nanjing, China). Mice ( $n=24,6$ weeks old) were housed three per cage in controlled environment with a constant temperature of $18{ }^{\circ} \mathrm{C}-22{ }^{\circ} \mathrm{C}$ and a humidity of $55 \%-60 \%$ on a 12:12-h light/dark cycle. After acclimation for seven days, mice were supplied with food and water freely every day. Mice were randomly divided into WT control, obese $\mathrm{db} / \mathrm{db}$ control, CL, and AS ( $n=6 /$ group) groups. Mice were immediately administered by oral gavage $100 \mathrm{mg} / \mathrm{kg} / \mathrm{day} \mathrm{CL}$ or $540 \mathrm{mg} / \mathrm{kg} /$ day AS, and the WT and obese $\mathrm{db} / \mathrm{db}$ groups received the same volume of distilled water for eight weeks. The food intake and body weight of the mice were measured weekly during the experimental period. CL is a long acting $\beta-2$ adrenergic agonist. In addition, CL, with a recommended dose of 20 to $40 \mu \mathrm{g}$ orally, is used as a bronchodilator in humans in Europe and Latin America [18]. Therefore, we used CL to compare with AS because its mechanism is very similar to AS in reducing weight losses. In other words, they both reduce body fat and increase basal lipolysis through increasing energy expenditure.

\subsection{Abdominal Computed Tomography Analysis}

Mice were under 4\% chloral hydrate in anesthesia and experimented for abdominal computed tomography analysis through micro-computed tomography (micro-CT). Mice were performed with an animal positron emission tomography (PET)/CT/single photon emission computed tomography (SPECT) system (INVEON, Siemens, Knoxville, TN, USA). The computed tomography pictures of mice were analyzed and processed using Siemen's Inveon software to calculate the volume of the fat mass between lumbar vertebrae one to five.

\subsection{Liver Lipid Content}

The determination of liver lipid content were measured and extracted with $\mathrm{CHCl}_{3}$-methanol (1:1) according to the method of Folch et al. [19]. $100 \mathrm{mg}$ of liver tissue was homogenized in $0.2 \mathrm{~mL}$ of phosphate buffer ( $\mathrm{pH}$ 7.4) using an ultrasonic irradiation (SONICS, Newtown, CT, USA). Lipids were extracted by mixing with lysate sample and $\mathrm{CHCl}_{3}$-methanol. Subsequently, the homogenates were subjected to assays for lipid content using commercially available kits.

\subsection{Histological Analysis}

For the morphology examination, mice were sacrificed by an intraperitoneal injection of $4 \%$ chloral hydrate at the eighth week. The same section of liver and epididymis adipose tissue were fixed with $10 \%$ buffered formalin for one day. The tissue was embedded in paraffin after dehydration in ethanol, and cut into sections at a thickness of $5 \mu \mathrm{m}$, which were stained with hematoxylin and eosin. In addition, Oil Red-O staining was performed in frozen liver sections to detect the lipid droplets in the hepatocytes. One frozen section of liver tissue was cut into for $5 \mu \mathrm{m}$, which were stained with Oil Red-O. Images at $200 \times$ magnification were photographed using an IX71 inverted light microscope (Olympus, Tokyo, Japan).

\subsection{Biochemical Parameters Analysis}

At the end of the experiment, mice were anesthetized and sacrificed after an overnight fast. Serum was collected from the abdominal vena cava and separated by centrifugation at $1000 \mathrm{~g}$ for $10 \mathrm{~min}$ at room temperature. Serum TG, FFA, glucose, cholinesterase (CHE), AST, and ALT levels were measured using a Model 7180 automated biochemical analyzer (Hitachi, Tokyo, Japan), according to the manufacturer's instructions. Inflammatory cytokine levels were quantified using ELISA kits 
specific for mouse serum TNF- $\alpha$, IL-1 $\beta$, and IL-6 levels, following the protocol recommended by the manufacturer.

\subsection{Western Blot Analysis}

Total protein was collected from tissues with lysis buffer containing a protease inhibitor cocktail followed by centrifugation at $12,000 \mathrm{rpm}$ for $15 \mathrm{~min} 4{ }^{\circ} \mathrm{C}$. Protein concentration was determined using a bicinchoninic acid kit. Equal amounts of proteins were electrophoresed by $6 \%$ or $8 \%$ sodium dodecyl sulfate polyacrylamide gel electrophoresis and then transferred onto polyvinylidene difluoride membranes (Millipore, Billerica, MA, USA). The membranes were blocked with 5\% skim milk powder in tris-buffered saline with Tween 20 (TBST; 0.1\% Tween 20, $20 \mathrm{mM}$ Tris-base, $150 \mathrm{mM} \mathrm{NaCl}$, $\mathrm{pH}$ 7.5) for $2 \mathrm{~h}$. The membranes were then incubated overnight at $4{ }^{\circ} \mathrm{C}$ with primary antibodies against perilipin, ATGL and HSL, SREBP-1, FAS and HMGCR (1:1000). After washing three times with TBST, membranes were incubated with a secondary HRP-conjugated goat anti-rabbit IgG (1:1000) for $1.5 \mathrm{~h}$. $\beta$-actin or GAPDH antibody (1:4000) was used as a loading control. Protein bands were visualized using enhanced chemiluminescence (Beyotime, Shanghai, China).

\subsection{Statistical Analysis}

All data were carried out using Graph Pad Prism v.5.0 for Windows (GraphPad, La Jolla, CA, USA). The results are expressed as mean \pm SEM. Differences between groups were performed with the one-way analysis of variance (ANOVA), followed by a post hoc comparison with the Bonferroni test. The data were considered statistically significant at $p$-values $<0.05$.

\section{Discussion}

Obesity has become an international public health problem that affects people quality of life and is associated with various kinds of diseases. NAFLD undergoes an upward trend with the increased prevalence and incidence of obesity and T2DM [20]. Some anti-obesity drugs have been approved by the U.S. Food and Drug Administration for chronic obesity management. However, most anti-obesity drugs have been limited by their various side effects in the clinic. Orlistat, a lipase inhibitor, reduces weight by $3 \mathrm{~kg}$ by inhibiting gastrointestinal lipases; however, it is associated with hepatotoxicity and adverse gastrointestinal effects [21]. Sibutramine, a norepinephrine and serotonin reuptake inhibitor, reduces weight by $4-5 \mathrm{~kg}$ on average by suppressing appetite, but it may increase the risk of mania or panic, psychosis, and cardiovascular diseases [22]. CL, a long acting beta-2 adrenergic agonist, has been shown to reduce body fat and increase basal lipolysis through increasing energy expenditure in mice [23]. However, it was accompanied by cardiac diseases and hypokalemia [24]. However, anti-obesity has unique $\beta_{3}$-adrenergic effects of $C L$, unrelated to its brochodialating effects, which has helped CL reduce body fat and increase basal lipolysis. Many studies have elaborated that CL increases adipocyte lipolysis, glycolytic capacity, and minimizes protein degradation in animals and humans. We used CL to compare with AS because its mechanism is very similar to AS in reducing weight losses. In other words, they both reduce body fat and increase basal lipolysis through increasing energy expenditure. However AS is an efficient anti-obesity drug from natural sources, unlike drugs above with seriously side effects. Up to now, many studies have demonstrated the anti-tumor, anti-bacterial, anti-viral, anti-inflammatory, and wound healing effects of Zicao $[15,25,26]$; our study indicates other important pharmacologic effects of Zicao.

In the present study, we investigate the anti-obesity and anti-NAFLD effects of AS in spontaneous obese $\mathrm{db} / \mathrm{db}$ mice. Eight weeks administration of AS significantly reduces body weight gain, fat tissue, and liver weight without affecting food intake. There is a significant reduction in abdominal fat mass and BMI in the AS group compared with that of the obese $\mathrm{db} / \mathrm{db}$ group. A study has been reported that the prevalence rate of NAFLD is accompanied with increasing BMI [27]. Both abdominal fat mass and BMI are the increased risk factors for NAFLD, T2DM, and cardiovascular diseases [28]. In addition, our studies also provide evidence that AS inhibits hepatic lipid droplet accumulation. 
Moreover, serum FFA, TG, AST, and ALT levels, which were elevated in $\mathrm{db} / \mathrm{db}$ mice, were decreased by AS treatment. In addition, the levels of serum TNF- $\alpha$, IL-1 $\beta$ and IL- 6 are increased in $\mathrm{db} / \mathrm{db}$ mice compared with that of WT mice, whereas treatment of AS markedly suppressed the increase in the levels of inflammatory cytokines. These results indicate that AS can ameliorate the symptoms of obesity and NAFLD.

Obesity is a complex metabolic syndrome associated with NAFLD [29]. Many studies have found that lipolysis may play important roles in regulating obesity and related metabolic disorders such as fatty liver disease and diabetes mellitus [30]. Adipocyte lipolysis is through increasing in intracellular cyclic AMP levels, which activates the activation of protein kinase A and then enhances the expression of downstream molecules HSL, ATGL and perilipin, as a result of the release of FFA and glycerol from TG [31,32]. We evaluated the effect of AS treatment on the expression of HSL, ATGL and perilipin in adipose tissue by Western blotting, which demonstrated that AS can increase lipolysis by upregulating the expression of perilipin, ATGL and HSL in adipose tissue.

NAFLD develops and progresses to severe liver diseases, coronary atherosclerotic heart disease, and acute pancreatitis [33]. Lipid metabolism contains lipolysis and lipid synthesis. SREBP-1, a gene encoding of hepatic transcription, regulates fatty acid metabolism and lipid synthesis in the liver. In addition, HMGCR, a crucial enzyme of cholesterol synthesis, is involved in fatty acid metabolism and regulates serum FFA level. Furthermore, SREBP-1 also regulates the expression of enzymes (such as acetyl-CoA carboxylase and FAS) involved in mediating hepatic synthesis of fatty acids, TGs and cholesterol [34]. These cascades eventually increase fat accumulation within hepatocytes due to the amount of fatty acids entering the liver. In this study, we showed that the treatment $\mathrm{db} / \mathrm{db}$ mice with AS resulted in a decrease of SREBP-1, FAS and HMGCR lipoprotein levels. Collectively, these findings suggest that AS exerts the treatment of NAFLD through regulating hepatic lipid and lipoprotein metabolism.

With the rising incidence of obesity worldwide, NAFLD has become a national public health concern. Moreover the pathogenesis of NAFLD has not been fully elucidated. A 'two-hit' hypothesis regarding the complex pathogenetic mechanism of NAFLD/NASH has traditionally been proposed. The 'first hit' is associated with reversible deposition of TGs within hepatocytes; however, the 'second hit' is in combination with the generation of free radicals, oxidative stress reaction and the production of inflammatory cytokines that progresses eventually to liver fibrosis [35]. NAFLD pathophysiologic processes linking obesity enhanced circulating concentrations of pro-inflammatory cytokines and factors (e.g., TNF- $\alpha$, IL-6 and IL-1 $\beta$ ). TNF- $\alpha$ and IL-1 $\beta$, pro-inflammatory and lipogenic factors, play crucial roles in the pathogenesis of NAFLD/NASH (liver steatosis, necrosis, apoptosis and fibrosis) [36]. IL-6, another pro-inflammatory cytokine, has been presented to directly and indirectly injure hepatocytes and induce inflammation [37]. The above pro-inflammatory cytokines provoke the production of other cytokines and then recruit inflammatory cells, resulting in liver injury and hepatic fibrosis; however, they are barely found in the serum of healthy mice [38]. Therefore, in our study, TNF- $\alpha$, IL- 6 and IL- $1 \beta$ were marked as representative inflammatory factors involved in the inflammation of liver. We demonstrated that the treatment of $\mathrm{db} / \mathrm{db}$ mice with AS resulted in a decrease of TNF- $\alpha$, IL- 6 and IL-1 levels. Taken together, AS exerts the effective protection against the development of hepatic steatosis, which may be due to the anti-inflammatory property.

\section{Conclusions}

Obesity and NAFLD are considered important parts of the metabolic syndrome worldwide. The herbal compound of AS is appeared to be an effective therapeutic agent that exerts effective protection against the obesity and NAFLD in a model of spontaneous obese $\mathrm{db} / \mathrm{db}$ mice by the regulation of lipid metabolism and anti-inflammatory effect. Further studies focusing on the effects of AS on liver fibrosis are required. 
Acknowledgments: The authors thank Menglan Chen (The First Affiliated Hospital, Sun Yat-sen University, and Guangzhou, China) for kindly analyzing the mouse abdominal computed tomography. This research did not receive any specific grants from funding agencies in the public, commercial, or not-for-profit sectors.

Author Contributions: Mei-Ling Su and Bang-Hao Zhu participated in research design; Mei-Ling Su and Bang-Hao Zhu conducted experiments; Mei-Ling Su; Yu He, and Qi-Sen Li contributed new reagents or analytic tools; Mei-Ling Su and Yu He performed data analysis; Mei-Ling Su; Yu He; Qi-Sen Li; Bang-Hao Zhu wrote or contributed to the writing of the manuscript.

Conflicts of Interest: The authors declare no conflict of interest.

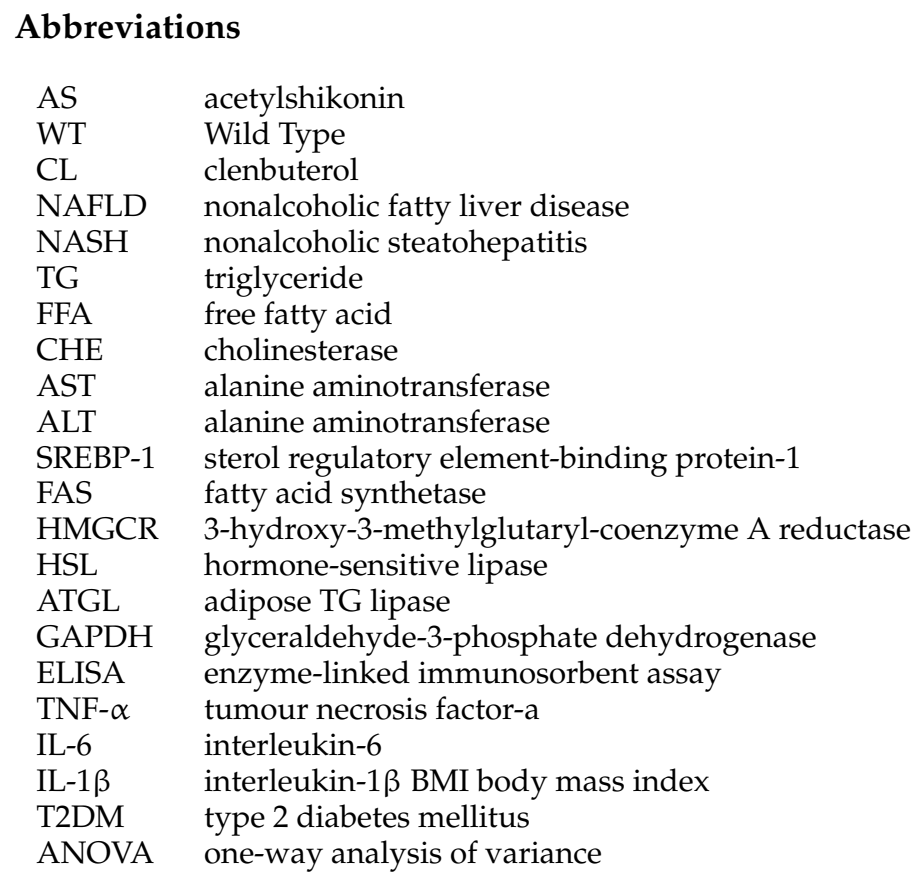

\section{References}

1. Kyrou, I.; Randeva, H.S.; Weickert, M.O. Clinical Problems Caused by Obesity. In Endotext (Internet); MDText.com, Inc.: Dartmouth, MA, USA, 2000.

2. Williams, K.H.; Shackel, N.A.; Gorrell, M.D.; McLennan, S.V.; Twigg, S.M. Diabetes and nonalcoholic Fatty liver disease: A pathogenic duo. Endocr. Rev. 2013, 34, 84-129. [CrossRef] [PubMed]

3. Smith, B.W.; Adams, L.A. Nonalcoholic fatty liver disease and diabetes mellitus: Pathogenesis and treatment. Nat. Rev. Endocrinol. 2011, 7, 456-465. [CrossRef] [PubMed]

4. Kaswala, D.H.; Lai, M.; Afdhal, N.H. Fibrosis Assessment in Nonalcoholic Fatty Liver Disease (NAFLD) in 2016. Dig. Dis. Sci. 2016, 61, 1356-1364. [CrossRef] [PubMed]

5. Kratky, D.; Obrowsky, S.; Kolb, D.; Radovic, B. Pleiotropic regulation of mitochondrial function by adipose triglyceride lipase-mediated lipolysis. Biochimie 2014, 96, 106-112. [CrossRef] [PubMed]

6. Morak, M.; Schmidinger, H.; Riesenhuber, G.; Rechberger, G.N.; Kollroser, M.; Haemmerle, G.; Zechner, R.; Kronenberg, F.; Hermetter, A. Adipose triglyceride lipase (ATGL) and hormone-sensitive lipase (HSL) deficiencies affect expression of lipolytic activities in mouse adipose tissues. Mol. Cell. Proteom. 2012, 11, 1777-1789. [CrossRef] [PubMed]

7. Wang, Y.; Li, J.Y.; Han, M.; Wang, W.L.; Li, Y.Z. Prevention and treatment effect of total flavonoids in Stellera chamaejasme L. on nonalcoholic fatty liver in rats. Lipids Health Dis. 2015, 14. [CrossRef] [PubMed]

8. Moon, Y.A.; Liang, G.; Xie, X.; Frank-Kamenetsky, M.; Fitzgerald, K.; Koteliansky, V.; Brown, M.S.; Goldstein, J.L.; Horton, J.D. The Scap/SREBP pathway is essential for developing diabetic fatty liver and carbohydrate-induced hypertriglyceridemia in animals. Cell Metab. 2012, 15, 240-246. [CrossRef] [PubMed]

9. Devisscher, L.; Verhelst, X.; Colle, I.; van Vlierberghe, H.; Geerts, A. The role of macrophages in obesity-driven chronic liver disease. J. Leukoc. Biol. 2016, 99, 693-698. [CrossRef] [PubMed] 
10. Lanthier, N.; Molendi-Coste, O.; Cani, P.D.; van Rooijen, N.; Horsmans, Y.; Leclercq, I.A. Kupffer cell depletion prevents but has no therapeutic effect on metabolic and inflammatory changes induced by a high-fat diet. FASEB J. 2011, 25, 4301-4311. [CrossRef] [PubMed]

11. Tilg, H.; Moschen, A.R.; Szabo, G. Interleukin-1 and inflammasomes in ALD/AAH and NAFLD/NASH. Hepatology 2016. [CrossRef] [PubMed]

12. Moschen, A.R.; Kaser, S.; Tilg, H. Non-alcoholic steatohepatitis: A microbiota-driven disease. Trends Endocrinol. Metab. 2013, 24, 537-545. [CrossRef] [PubMed]

13. Tsochatzis, E.A.; Papatheodoridis, G.V.; Archimandritis, A.J. Adipokines in nonalcoholic steatohepatitis: From pathogenesis to implications in diagnosis and therapy. Mediat. Inflamm. 2009, 2009. [CrossRef] [PubMed]

14. Chen, X.; Yang, L.; Oppenheim, J.J.; Howard, M.Z. Cellular pharmacology studies of shikonin derivatives. Phytother. Res. 2002, 16, 199-209. [CrossRef] [PubMed]

15. Zeng, Z.; Zhu, B.H. Arnebin-1 promotes the angiogenesis of human umbilical vein endothelial cells and accelerates the wound healing process in diabetic rats. J. Ethnopharmacol. 2014, 154, 653-662. [CrossRef] [PubMed]

16. Andujar, I.; Rios, J.L.; Giner, R.M.; Recio, M.C. Pharmacological properties of shikonin-A review of literature since 2002. Planta Med. 2013, 79, 1685-1697. [CrossRef] [PubMed]

17. Su, M.; Huang, W.; Zhu, B. Acetylshikonin from Zicao Prevents Obesity in Rats on a High-Fat Diet by Inhibiting Lipid Accumulation and Inducing Lipolysis. PLoS ONE 2016, 11, e146884. [CrossRef] [PubMed]

18. Thompson, J.A.; Mirza, M.H.; Barker, S.A.; Morgan, T.W.; Bauer, R.W.; McConnico, R.S. Clenbuterol toxicosis in three Quarter Horse racehorses after administration of a compounded product. J. Am. Vet. Med. Assoc. 2011, 239, 842-849. [CrossRef] [PubMed]

19. Folch, J.; Lees, M.; Sloane, S.G. A simple method for the isolation and purification of total lipides from animal tissues. J. Biol. Chem. 1957, 226, 497-509. [PubMed]

20. Chalasani, N.; Younossi, Z.; Lavine, J.E.; Diehl, A.M.; Brunt, E.M.; Cusi, K.; Charlton, M.; Sanyal, A.J. The diagnosis and management of non-alcoholic fatty liver disease: Practice Guideline by the American Association for the Study of Liver Diseases, American College of Gastroenterology, and the American Gastroenterological Association. Hepatology 2012, 55, 2005-2023. [CrossRef] [PubMed]

21. Leung, W.Y.; Thomas, G.N.; Chan, J.C.; Tomlinson, B. Weight management and current options in pharmacotherapy: Orlistat and sibutramine. Clin. Ther. 2003, 25, 58-80. [CrossRef]

22. Padwal, R.S.; Majumdar, S.R. Drug treatments for obesity: Orlistat, sibutramine, and rimonabant. Lancet 2007, 369, 71-77. [CrossRef]

23. Kim, H.K.; Della-Fera, M.A.; Hausman, D.B.; Baile, C.A. Effect of clenbuterol on apoptosis, adipogenesis, and lipolysis in adipocytes. J. Physiol. Biochem. 2010, 66, 197-203. [CrossRef] [PubMed]

24. Yen, M.; Ewald, M.B. Toxicity of weight loss agents. J. Med. Toxicol. 2012, 8, 145-152. [CrossRef] [PubMed]

25. Ozaki, Y.; Ohno, A.; Saito, Y.; Satake, M. Accelerative effect of shikonin, alkannin and acetylshikonin on the proliferation of granulation tissue in rats. Biol. Pharm. Bull. 1994, 17, 1075-1077. [CrossRef] [PubMed]

26. Kuo, H.M.; Hsia, T.C.; Chuang, Y.C.; Lu, H.F.; Lin, S.Y.; Chung, J.G. Shikonin inhibits the growth and $\mathrm{N}$-acetylation of 2-aminofluorene in Helicobacter pylori from ulcer patients. Anticancer Res. 2004, 24, 1587-1592. [PubMed]

27. Ruhl, C.E.; Everhart, J.E. Determinants of the association of overweight with elevated serum alanine aminotransferase activity in the United States. Gastroenterology 2003, 124, 71-79. [CrossRef] [PubMed]

28. Wronska, A.; Kmiec, Z. Structural and biochemical characteristics of various white adipose tissue depots. Acta Physiol. (Oxf.) 2012, 205, 194-208. [CrossRef] [PubMed]

29. Fabbrini, E.; Sullivan, S.; Klein, S. Obesity and nonalcoholic fatty liver disease: Biochemical, metabolic, and clinical implications. Hepatology 2010, 51, 679-689. [CrossRef] [PubMed]

30. Fabbrini, E.; Magkos, F. Hepatic Steatosis as a Marker of Metabolic Dysfunction. Nutrients 2015, 7, 4995-5019. [CrossRef] [PubMed]

31. Carmen, G.Y.; Victor, S.M. Signalling mechanisms regulating lipolysis. Cell Signal. 2006, 18, 401-408. [CrossRef] [PubMed]

32. Holm, C. Molecular mechanisms regulating hormone-sensitive lipase and lipolysis. Biochem. Soc. Trans. 2003, 31, 1120-1124. [CrossRef] [PubMed] 
33. Semiserin, V.A.; Karakozov, A.G.; Mal'Kuta, M.A.; Zolotareva, L.A.; Levchenko, O.B.; Kalyagin, I.E.; Eremin, M.N. Evaluation of the efficiency of hepatoprotective monotherapy using succinic acid and methionine for nonalcoholic fatty liver disease at the stage of steatohepatitis. Ter Arkh 2016, 88, 58-63. [CrossRef] [PubMed]

34. Ameer, F.; Scandiuzzi, L.; Hasnain, S.; Kalbacher, H.; Zaidi, N. De novo lipogenesis in health and disease. Metabolism 2014, 63, 895-902. [CrossRef] [PubMed]

35. Day, C.P.; James, O.F. Steatohepatitis: A tale of two "hits"? Gastroenterology 1998, 114, 842-845. [CrossRef]

36. Inzaugarat, M.E.; Ferreyra, S.N.; Billordo, L.A.; Abecasis, R.; Gadano, A.C.; Chernavsky, A.C. Altered phenotype and functionality of circulating immune cells characterize adult patients with nonalcoholic steatohepatitis. J. Clin. Immunol. 2011, 31, 1120-1130. [CrossRef] [PubMed]

37. Braunersreuther, V.; Viviani, G.L.; Mach, F.; Montecucco, F. Role of cytokines and chemokines in non-alcoholic fatty liver disease. World J. Gastroenterol. 2012, 18, 727-735. [CrossRef] [PubMed]

38. Tilg, H.; Hotamisligil, G.S. Nonalcoholic fatty liver disease: Cytokine-adipokine interplay and regulation of insulin resistance. Gastroenterology 2006, 131, 934-945. [CrossRef] [PubMed]

Sample Availability: Samples of the compounds are available from the authors.

(C) 2016 by the authors; licensee MDPI, Basel, Switzerland. This article is an open access article distributed under the terms and conditions of the Creative Commons Attribution (CC-BY) license (http:/ / creativecommons.org/licenses/by/4.0/). 